\title{
SOLAR NEUTRINOS. II. EXPERIMENTAL*
}

\author{
Raymond Davis, Jr. \\ Chemistry Department, Brookhaven National Laboratory, Upton, New York \\ (Received 6 January 1964)
}

The prospect of observing solar neutrinos by means of the inverse beta process ${ }^{37} \mathrm{Cl}\left(\nu, e^{-}\right)^{37} \mathrm{Ar}$ induced us to place the apparatus previously described $^{1}$ in a mine and make a preliminary search. This experiment served to place an upper limit on the flux of extraterrestrial neutrinos. These results will be reported, and a discussion will be given of the possibility of extending the sensitivity of the method to a degree capable of measuring the solar neutrino flux calculated by Bahcall in the preceding paper. ${ }^{2}$

The apparatus consists of two 500-gallon tanks of perchlorethylene, $\mathrm{C}_{2} \mathrm{Cl}_{4}$, equipped with agitators and an auxiliary system for purging with helium. It is located in a limestone mine 2300 feet below the surface ${ }^{3}$ (1800 meters of water equivalent shielding, m.w.e.). Initially the tanks were swept completely free of air argon by purging the tanks with a stream of helium gas. ${ }^{36} \mathrm{Ar}$ carrier $\left(0.10 \mathrm{~cm}^{3}\right)$ was introduced and the tanks exposed for periods of four months or more to allow the $35-\mathrm{d}{ }^{37} \mathrm{Ar}$ activity to reach nearly the saturation value. Carrier argon along with any ${ }^{37} \mathrm{Ar}$ produced were removed from the tanks by sweeping them in series with 5000 liters of helium. Argon was extracted from the helium gas stream with activated charcoal at $78^{\circ} \mathrm{K}$. Finally the argon was desorbed from the charcoal, purified and counted. The over-all efficiency of the processing was determined by ${ }^{36} \mathrm{Ar}$ isotopic analysis of the recovered argon. The recovery of carrier argon was always greater than $95 \%$. The entire argon sample was placed in a small proportional counter $1.2 \mathrm{~cm}$ long and $0.3 \mathrm{~cm}$ in diameter to measure the ${ }^{37} \mathrm{Ar}$ activity. Pulse-height analysis was used, and counters were recorded in anticoincidence with a ring of proportional counters, and an enveloping NaI crystal. The counter was provided with an end window to permit exposure of the counting volume to ${ }^{55} \mathrm{Fe}$ $x$ rays for energy calibration and determination of the resolution of the counter. The resolution, full width at half-height for the 2.8-keV Auger electrons from the ${ }^{37} \mathrm{Ar}$ decay, was $26 \%$. The over-all counter efficiency for ${ }^{37} \mathrm{Ar}$ in the full peak was $46 \%$. The counting rate with the sample was 3 counts in 18 days and this is the same as the background rate for the counter filled with air argon. Therefore the observed counting rate of
3 counts in 18 days is probably entirely due to the background activity. However, if one assumes that this rate corresponds to real events and uses the efficiencies mentioned, the upper limit of the neutrino capture rate in 1000 gallons of $\mathrm{C}_{2} \mathrm{Cl}_{4}$ is $\leqslant 0.5$ per day or $\varphi \bar{\sigma} \leqslant 3 \times 10^{-34} \mathrm{sec}^{-1}\left({ }^{37} \mathrm{Cl} \text { atom }\right)^{-1}$. From this value, Bahcall ${ }^{2}$ has set an upper limit on the central temperature of the sun and other relevant information.

On the other hand, if one wants to measure the solar neutrino flux by this method one must use a much larger amount of $\mathrm{C}_{2} \mathrm{Cl}_{4}$, so that the expected ${ }^{37} \mathrm{Ar}$ production rate is well above the background of the counter, 0.2 count per day. Using Bahcall's expression,

$$
\begin{aligned}
& \sum \varphi_{\nu}(\text { solar }) \sigma_{\text {abs }} \\
& \quad=(4 \pm 2) \times 10^{-35} \mathrm{sec}^{-1}\left({ }^{37} \mathrm{Cl} \text { atom }\right)^{-1}
\end{aligned}
$$

then the expected solar neutrino captures in 100000 gallons of $\mathrm{C}_{2} \mathrm{Cl}_{4}$ will be 4 to 11 per day, which is an order of magnitude larger than the counter background. On the basis of experience obtained with the present experiment, an increase in the volume of liquid to 100000 gallons would not present any insuperable difficulties. The result of such an experiment would provide a valid test for the present theory of the solar energy generation process. The important features of the method are that small amounts of ${ }^{37} \mathrm{Ar}$ can be removed efficiently from large volumes of liquid by the simple procedure of sweeping with helium and that the characteristic decay of ${ }^{37} \mathrm{Ar}$ can be observed in a counter with an essentially zero background. There are, however, a number of other processes that could produce ${ }^{37} \mathrm{Ar}$ at these low levels in a tank of perchlorethylene in an underground mine; these other effects constitute an undesirable background. Alvarez ${ }^{4}$ made a thorough analysis of these unwanted effects in his original proposal some years ago. In general, background effects may arise from cosmic-ray muons, from fast neutrons from the surrounding rock wall, and from nuclear reactions arising from internal contaminations in the liquid.

Cosmic-ray background effects underground arise by the ${ }^{37} \mathrm{Cl}(p, n){ }^{37} \mathrm{Ar}$ reaction from the protons produced in muon interactions. The magni- 
Table I. ${ }^{37} \mathrm{Ar}$ production rates in $\mathrm{C}_{2} \mathrm{Cl}_{4}$ by cosmic-ray muons underground.

\begin{tabular}{cccc}
\hline \hline $\begin{array}{c}\text { Depth below } \\
\text { surface } \\
(\mathrm{m} . \mathrm{w} . \mathrm{e})\end{array}$ & $\begin{array}{c}\text { Muon intensity } \\
\left(\mathrm{cm}^{-2} \mathrm{sec}^{-1} \mathrm{sr}^{-1}\right)\end{array}$ & $\begin{array}{c}\text { Muon star production } \\
\text { cross section } \\
\left(\mathrm{cm}^{2} / \text { nucleon }\right)\end{array}$ & $\begin{array}{c}{ }^{37} \text { Ar production } \\
\text { rate per day for } \\
10^{5} \text { gallons } \mathrm{C}_{2} \mathrm{Cl}_{4}\end{array}$ \\
\hline 25 & $2 \times 10^{-3}$ & $3 \times 10^{-30}$ & 6500 (measured) \\
1800 & $2 \times 10^{-7}$ & $17 \times 10^{-30}$ & 3.5 \\
4000 & $6 \times 10^{-9}$ & $22 \times 10^{-30}$ & 0.14 \\
\hline \hline
\end{tabular}

tude of this background effect can be calculated from measurements made at a depth of $25 \mathrm{~m}$.w. e. ${ }^{5}$ where the nucleonic component is essentially eliminated, and the ${ }^{37} \mathrm{Ar}$ is produced by muons. At this depth a ${ }^{37} \mathrm{Ar}$ production rate of 210 atoms per day was observed 3000 gallons of $\mathrm{CCl}_{4}$. $\mathrm{Be}-$ low this depth the ${ }^{37} \mathrm{Ar}$ production should decrease with the product of the muon intensity and the cross section for star production by muons. Table I lists the muon intensities ${ }^{6}$ and cross sections ${ }^{7}$ used to calculate the ${ }^{37} \mathrm{Ar}$ production by muons at 1800 and $4000 \mathrm{~m} . \mathrm{w} . \mathrm{e}$.

It may be noted from Table I that the calculated rate at $1800 \mathrm{~m}$. w. e. is below the limit set by the present 1000-gallon experiment. However, it is clear that a large-scale experiment would have to be performed at a much greater depth. If the proposed experiment were conducted in a mine approximately 4500 feet deep ( $4000 \mathrm{~m}$.w. e. ) the muon-produced ${ }^{37} \mathrm{Ar}$ would be a factor of 30 below the expected rate of 4 to 11 per day from solar neutrinos.

${ }^{37} \mathrm{Ar}$ may also be produced in the liquid by energetic neutrons. Neutrons having an energy above $0.97 \mathrm{MeV}$ will produce protons by the exothermic ${ }^{35} \mathrm{Cl}(n, p){ }^{35} \mathrm{~S}$ reaction with sufficient energy to produce ${ }^{37} \mathrm{Ar}$ by the ${ }^{37} \mathrm{Cl}(p, n){ }^{37} \mathrm{Ar}$ reaction. This ef fect was evaluated by irradiating the liquid with a $\mathrm{Pu}-\mathrm{Be}$ neutron source. These measurements gave a yield of one ${ }^{37} \mathrm{Ar}$ atom per $1.4 \times 10^{6}$ neutrons absorbed. Fast neutrons from the surrounding rock could produce one ${ }^{37} \mathrm{Ar}$ atom per day if the neutron flux on the surface of the 100000 -gallon tank (26 ft diameter $\times 26 \mathrm{ft}$ high) were $4 \times 10^{-4}$ neutron $\mathrm{cm}^{-2} \mathrm{~min}^{-1}$. The fast neutron flux may be kept below this value by a water shield, the thickness depending on the uranium and thorium content of the rock wall.

Internal contaminations leading to ${ }^{37} \mathrm{Ar}$ production in the materials of the tank or the liquid itself cannot be shielded out, and would serve as an inherent background that could not be separated from a neutrino signal. We have, however, found that the thorium and uranium content of perchlor- ethylene was less than $2 \times 10^{-9} \mathrm{~g}$ per gram. At this level internal neutron production is unimportant; less than $0.01{ }^{37} \mathrm{Ar}$ would be produced per day by these neutrons. However, even at this uranium and thorium level the sulfur content must be below $0.5 \%$ to reduce the ${ }^{37} \mathrm{Ar}$ produced by the ${ }^{34} \mathrm{~S}(\alpha, n){ }^{37} \mathrm{Ar}$ reaction to less than one per day.

We may conclude from the above considerations that an experiment using 100000 gallons of pure perchlorethylene in a mine 4500 feet deep, properly shielded from fast neutrons, would have a background ${ }^{37} \mathrm{Ar}$ production rate at least a factor of ten below the expected rate from solar neutrinos. It should be noted that if a positive result were obtained from such an experiment there would remain a small ambiguity in interpretation because of the possibility of a galactic source of neutrinos. A possible method of distinguishing between solar and galactic neutrinos would be to take advantage of the eccentricity of the earth's orbit and measure the $7 \%$ difference in solar neutrino intensity between aphelion and perihelion. With a signal as low as 7 per day (a total of 350 ${ }^{37} \mathrm{Ar}$ atoms) such an experiment would be marginal, but if a somewhat higher signal was observed such a test would be possible.

Dr. John Bahcall, Dr. R. L. Sears, and Professor W. A. Fowler of the California Institute of Technology have provided much of the theoretical and experimental information that makes this experiment meaningful. I would like to thank them for keeping me informed of these developments. Also, I would like to acknowledge the assistance of Robert L. Chase for designing the low-background counting system.

\footnotetext{
*Research performed under the auspices of the U. S. Atomic Energy Commission.

${ }^{1} \mathrm{R}$. Davis, Jr., in Radioisotopes in Scientific Research (Pergamon Press, New York, 1958), Vol. 1.

${ }^{2} \mathrm{~J}$. Bahcall, preceding Letter [Phys. Rev。 Letters $12,300(1964)]$. If only ground-state transitions are considered, a solar neutrino capture rate in $10^{5}$ gallons of $\mathrm{C}_{2} \mathrm{Cl}_{4}$ from ${ }^{7} \mathrm{Be}$ and ${ }^{8} \mathrm{~B}$ neutrinos would be 0.6 and
} 
0.3 per day. An additional contribution to the rate of 5.7 per day would be expected from ${ }^{8} \mathrm{~B}$ neutrinos captured to form excited states in ${ }^{37} \mathrm{Ar}$ (approximately $50 \%$ error in flux). It is the contribution from excited states that produces an amount of ${ }^{37} \mathrm{Ar}$ in $10^{5}$ gallons of $\mathrm{C}_{2} \mathrm{Cl}_{4}$ well above that expected from background effects.

${ }^{3}$ The chemical division of the Pittsburgh Plate Glass Co. kindly allowed us to use their limestone mine at Barberton, Ohio, for this experiment. A more com- plete report will be published.

${ }^{4}$ L. W. Alvarez, University of California Radiation

Laboratory Report No. UCRL-328, 1949 (unpublished).

${ }^{5}$ R. Davis, Jr., and D. S. Harmer (to be published).

${ }^{6} \mathrm{P}$. H. Barrett, L. M. Bollinger, G. Cocconi,

Y. Eisenberg, and K. Greisen, Rev. Mod. Phys.

24, 133 (1952); J. Pine, R. J. Davisson, and K. Greisen, Nuovo Cimento 14, 1181 (1959).

${ }^{7}$ G. N. Fowler and A. W. Wolfendale, Progr. Elem. Particle Cosmic Ray Phys. $\underline{4}, 105$ (1958).

\section{3-PION DECAY OF THE INTERMEDIATE VECTOR BOSON*}

Mirza A. B. Bég, ${ }^{\dagger}$ John M. Cornwall, and Ching-Hung Woo Institute for Advanced Study, Princeton, New Jersey

(Received 10 February 1964)

In a recent Letter ${ }^{1}$ Feinberg and Mani calculated the branching ratio $R=\Gamma(W \rightarrow 3 \pi) / \Gamma(e+\nu)$ for a range of $m_{W}$ around $1.4 \mathrm{BeV}$, and found it to be comparable to unity. ${ }^{2}$ Such a large value for $R$ would considerably enhance its importance in the interpretation of current high-energy neutrino experiments; in particular one cannot estimate $m_{W}$ from observed reaction rates without a knowledge of $R$. It seems worthwhile, therefore, to have an independent estimate of $R$, based on considerations different from those implicit in reference 1. More specifically, we are motivated by the following reasons: (1) Feinberg and Mani used a value of $W \rho \pi$ coupling given in a paper by one of us ${ }^{3}$ (as a byproduct of a calculation of the nucleon axial-vector form factor). However, the considerations there are mainly concerned with the rate of variation of the form factor with momentum transfer, and are insensitive to the normalization at a particular value of momentum transfer. Hence the value of $W \rho \pi$ coupling deduced thereby may not be too reliable. (2) $W \rightarrow \rho+\pi$ involves two form factors and one of them was neglected in reference 1 . To be more confident of calculated decay rates one should have some knowledge of the relative magnitude of these form factors.

In the present note we use techniques familiar from a derivation of the Goldberger-Treiman formula. ${ }^{4}$ As a simple illustration we first consider $W \rightarrow \pi+\sigma$, where $\sigma$ is a scalar di-pion state ${ }^{5}$ $\left(T=0,0^{++}\right)$. Turning our attention to $W \rightarrow \pi+\rho$ we give a plausibility argument indicating that the form factor neglected in reference 1 is indeed small. An argument similar to that in the $\pi \sigma$ case then enables us to write down the $W \rho \pi$ coupling constant. Finally, in Table I, we tabulate the decay rates.

(1). Scalar di-pion state. - Let $A_{\mu}$ be the weak axial vector current. One can write

$$
\begin{aligned}
2\left(E_{\pi} E_{\sigma}\right)^{1 / 2}\left\langle\pi \sigma\left|A_{\mu}\right| 0\right\rangle & \\
= & {\left[\left(p_{\sigma}-p_{\pi}\right)_{\mu} f_{1}(s)+\left(p_{\sigma}+p_{\pi}\right)_{\mu} f_{2}(s)\right] P, } \\
& 2\left(E_{\pi} E_{\sigma}\right)^{1 / 2}\left\langle\pi \sigma\left|i \partial^{\mu} A_{\mu}\right| 0\right\rangle=h(s) P, \\
& \sqrt{2} E_{\pi}^{1 / 2}\left\langle\pi\left|A_{\mu}\right| 0\right\rangle=\left(p_{\pi}\right)_{\mu} a\left(m_{\pi}{ }^{2}\right) P,
\end{aligned}
$$

where $s=\left(p_{\pi}+p_{\sigma}\right)^{2}$ and $P \equiv \frac{1}{2}\left(\delta_{\alpha 1}+i \delta_{\alpha 2}\right)$ with $\alpha$ referring to the isotopic spin index of $\pi$.

The process $W \rightarrow \pi+\sigma$ is determined by the form factor $f_{1}$. For small $s$ the function $h(s)$ is dominated by the pion pole, and one has

$$
h(0)=g_{\pi \pi \sigma} a\left(m_{\pi}^{2}\right) .
$$

Using the Goldberger-Treiman formula for $a$, we find

$$
\left(m_{\sigma}^{2}-m_{\pi}^{2}\right) f_{1}(0)=h(0)=-g_{\pi \pi \sigma}\left(\frac{2 M}{g_{\pi N \bar{N}}}\right) G_{A},
$$

where $G_{A}$ is the axial vector coupling constant of $\beta$ decay, and $M=$ nucleon mass.

If we neglect rescattering corrections, we can take $f_{1}(0)$ to be the coupling constant effective in $W \rightarrow \pi+\sigma$. We then have

$$
\frac{\Gamma(W \rightarrow \sigma+\pi)}{\Gamma(W \rightarrow e+\nu)}=\frac{q_{\sigma}^{3}}{2 G_{V}{ }^{2} m_{W}{ }^{3}}\left|f_{1}(0)\right|^{2},
$$

$q_{\sigma}$ being the c.m. momentum of $\pi$ and $\sigma$.

For numerical orientation ${ }^{5}$ we take $m_{\sigma}{ }^{2} \approx 8 m_{\pi}{ }^{2}$, $\Gamma_{\sigma}=49 \mathrm{MeV}$. The result is summarized in col- 